\title{
High And Dehiscent Jugular Bulb in External Ear Canal: A Case Report
}

\author{
Isaac Montoya Hernández ${ }^{1 *}$ and Jesus Eduardo Cisneros Montelongo ${ }^{2}$ \\ ${ }^{1}$ Department of Otorhinolaryngology Head and Neck Surgery, Autonomous University of Coahuila, Mexico \\ ${ }^{2}$ Department of Otorhinolaryngology Head and Neck Surgery, Autonomous University of Coahuila, Mexico
}

*Corresponding author: Isaac Montoya Hernández, Department of Otorhinolaryngology Head and Neck Surgery, High Specialty Medical Unit/Specialty, Hospital 71, Mexican Institute of Social Security, Mexico.

Boulevard Revolución 2650 East. Col. Torreón Jardín. Torreón, 27200, Torreón, Coahuila, Mexico.
Received Date: October 19, 2021

Published Date: November 09, 2021

\begin{abstract}
The high jugular bulb is the common anatomical variation of the jugular bulb in the temporal bone. Encounters with jugular bulb abnormalities during ear surgery are a rare but recognized problem. The case of a 60 -year-old male with a subtotal dry eardrum perforation who underwent and elective tympanoplasty and unexpected brisk hemorrhage in external acoustic canal incisions is presented.
\end{abstract}

Keywords: Case report; Jugular bulb; Jugular bulb dehiscence; Temporal bone abnormality; Middle ear surgery; Tympanoplasty

Abbreviations: HJB: High jugular bulb; JB: Jugular bulb; CT: Computed tomography; ENT: Ears-nose-throat

\section{Introduction}

The jugular bulb is a dynamic structure that forms after 2 years and stabilizes in adulthood. The determinants of its exact position and size are multifactorial and likely are dependent on postnatal events [1]. The high jugular bulb (HJB), which indicates its superior (either medial or lateral) position to the cochlea, is the most common anatomical variation of the jugular bulb in the temporal bone [2]. This abnormality can be present in 10\%-15\% of individuals and can be dehiscent in $0.5 \%-1.7 \%[3,4]$. These variations are rarely symptomatic, presenting pulsatile tinnitus, conductive hearing loss and vertigo, depending on position [5-7]. Encounters with jugular bulb abnormalities during ear surgery are a rare but recognized problem [8-12]. The aim of this article is to describe an unexpected bleeding during a middle ear surgery, its management, radiological findings and discuss relevant literature.

\section{Case Presentation}

A 60-year-old male presented with four-year history of rightsided interment non pulsatile tinnitus, ipsilateral otalgia, and a mild conductive hearing loss, without any associated otorrhea, dizziness or facial

weakness. Examination of the right ear revealed a subtotal dry perforation of the tympanic membrane. Computed tomography scan of the patient's temporal bones demonstrated no signs of chronic process and structural anomalies were not reported. The patient was admitted for an elective tympanoplasty. During the surgery with postauricular approach, brisk venous bleeding was encountered at the point of the annulus upon removal of the skin of the anterior wall of the external acoustic canal. The hemorrhage 
arose from a dehiscent high riding jugular bulb lying immediately lateral to the annulus, in the pretympanic recess. Hemorrhage control was achieved through suction, extraluminal technique applying absorbent hemostatic gauze with hemostatic gelatin sponge, and replacing the anterior skin flap. Further review of the CT scan revealed a high, jugular bulb bulging into the external ear canal. Patient was discharged three days following procedure and still under tight follow up to monitor his condition (Figure 1, 2).

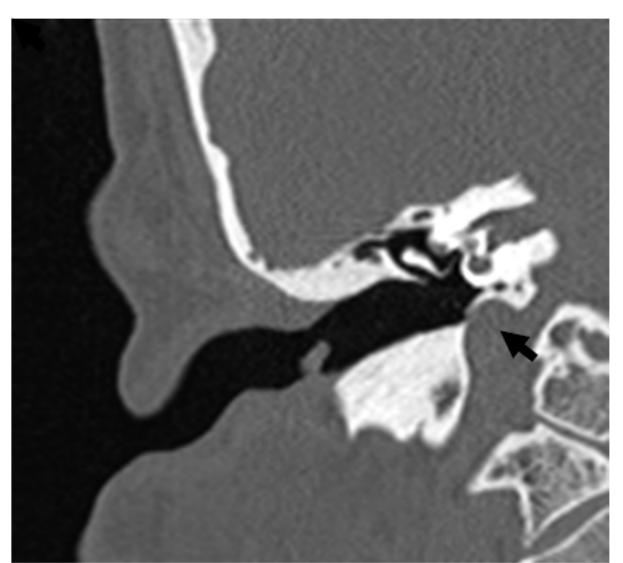

Figure 1: Coronal, high resolution computed tomography image of the right petrous temporal bone. Jugular bulb protruding in the tympanic recess (arrow).

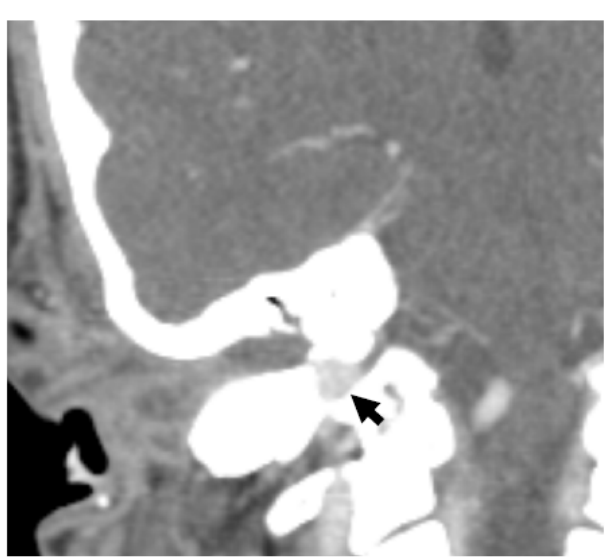

Figure 2: Coronal, venous phase of CT angiography image of the right petrous temporal bone after surgical act. Jugular bulb protruding in the tympanic recess and its dehiscence (arrow).

\section{Discussion}

Dehiscent jugular bulb is defined as a lack of bone over the jugular bulb, which occurs in $1-7 \%$ of bulb changes that affect the middle ear [13-15]. Although debatable, it seems that it also predominates on the right side, without differences regarding sex and being able to present in any age range $[1,13,14]$. In the clinical case presented, the location was right, though there was also an anormal presentation in contralateral temporal bone.

The anomalies of the jugular bulb are generally asymptomatic. However, the symptoms of suspected HJB may go unnoticed in the presence of a chronic otitis media process, as in our patient, and found directly in the surgery. If we have an imaging test, we must look for this type of alterations, because in general the jugular bulb area does not usually arouse the interest of the radiologist and the ENT, more pending the presence of cholesteatoma, position of the sigmoid sinus, tegmen tympani, ossicular chain, semicircular canals and facial nerve [4].
A HJB might be injured during surgical act. Case reports have described damage and resulting profuse hemorrhage as a result of myringotomy $[8,9]$, elevation of a tympanomeatal flap $[9,10,12]$, elevation of the inferior annulus [12], and removal of granulation tissue during middle-ear surgery [11]. Indeed, intrusion of a high jugular bulb into the middle ear has been well described. The only reported case of damage to a high jugular bulb during external ear canal surgery has been described by Moore [12]. In the clinical case presented, hemorrhage arose on removal of the skin of the anterior wall of the external acoustic canal, lateral to the annulus.

The management strategies in case of incidental surgical injury of a high and dehiscent JB, the postauricular approach is recommended to have a greater domain of the field3, as in our clinical case, in which the approach was originally performed for our planned procedure. When massive bleeding occurs during otologic surgery, it is recommended to place patient's head lower below the level of the heart to avoid air embolism and compression 
with gauze or absorbable material [9-12]. With this action, sometimes it is possible to complete the expected surgery, although the most frequent is, as in our current case, that it must be delayed after achieving hemostasis.

\section{Acknowledgment}

None.

\section{Conflict of Interest}

The authors declare that there is no conflict of interest.

\section{References}

1. Friedmann DR, Eubig J, McGill M, Babb JS, Pramanik BK, et al. (2011) Development of the jugular bulb: A radiologic study. Otol Neurotol 32(8): 1389-1395.

2. Wadin K, Wilbrand H (1986) The topographic relations of the high jugular fossa to the inner ear: A Radioanatomic Investigation. Acta Radiol Diagn (Stockh) 27(3): 315-324.

3. Huang BR, Wang CH, Young YH (2006) Dehiscent high jugular bulb: A pitfall in middle ear surgery. Otol Neurotol 27(7): 923-927.

4. Atmaca S, Elmali M, Kucuk H (2014) High and dehiscent jugular bulb: Clear and present danger during middle ear surgery. Surg Radiol Anat 36(4): 369-374.

5. Chennupati SK, Reddy NP, O'Reilly RC (2011) High-riding jugular bulb presenting as conductive hearing loss. International Journal of Pediatric Otorhinolaryngology Extra 6(4): 235-237.

6. Kizildag B, Bilal N, Yurttutan N, Sarica MA, Gungor G, et al. (2016) The relationship between tinnitus and vascular anomalies on temporal bone
CT scan: a retrospective case control study. Surg Radiol Anat 38(7): 835841.

7. DeHart AN, Shaia WT, Coelho DH (2018) Hydroxyapatite cement resurfacing the dehiscent jugular bulb: Novel treatment for pulsatile tinnitus. Laryngoscope 128(5): 1186-1190.

8. Page J (1914) A Case of Probable Injury to the Jugular Bulb following Myringotomy in an Infant Ten Months Old. Ann Otol Rhinol Laryngol 23(1):161-163.

9. Fox R, Nash R, Tatla T (2017) Encountering a high jugular bulb during ear surgery. Ann R Coll Surg Engl 99(1): e36-e37.

10. Tavárez Rodríguez J, Benito Orejas J, Fernández Rodríguez A, Mena Domínguez E, Hernández de los Santos M, et al. (2014) Jugular bulb bleeding in middle ear surgery. Caso clínico 5(27): 224-232.

11. Ball M, Elloy M, Vaidhyanath R, Pau H (2010) Beware the silent presentation of a high and dehiscent jugular bulb in the external ear canal. J Laryngol Otol 124(7): 790-792.

12. Moore PJ (1994) The high jugular bulb in ear surgery: Three case reports and a review of the literature. J Laryngol Otol 108(9): 772-775.

13. Woo CK, Wie CE, Park SH, Kong SK, Lee IW, et al. (2012) Radiologic analysis of high jugular bulb by computed tomography. Otol Neurotol 33(7): 1283-1287.

14. Kuhn MA, Friedmann DR, Winata LS, Jan Eubig, Bidyut K Pramanik, et al. (2012) Large jugular bulb abnormalities involving the middle ear. Otol Neurotol 33(7): 1201-1206.

15. Orr JB, Todd NW (1988) Jugular bulb position and shape are unrelated to temporal bone pneumatization. Laryngoscope 98(2): 136-138. 